\title{
TV/Series
}

$1 \mid 2012$

Les Séries télévisées américaines contemporaines :

entre la fiction, les faits, et le réel

\section{Lost in Lost : Entre quotidien anodin et déstabilisation fantastique, entre réalité alternative et fiction collective}

\section{Sarah Hatchuel}

\section{OpenEdition}

Journals

Édition électronique

URL : http://journals.openedition.org/tvseries/1569

DOI : 10.4000/tvseries.1569

ISSN : 2266-0909

Éditeur

GRIC - Groupe de recherche Identités et Cultures

Référence électronique

Sarah Hatchuel, «Lost in Lost : Entre quotidien anodin et déstabilisation fantastique, entre réalité alternative et fiction collective », TV/Series [En ligne], 1 | 2012, mis en ligne le 15 mai 2012, consulté le 19 avril 2019. URL : http://journals.openedition.org/tvseries/1569 ; DOI : 10.4000/tvseries.1569

\section{(c) $($ ) $(3)$}

TV/Series est mis à disposition selon les termes de la licence Creative Commons Attribution - Pas d'Utilisation Commerciale - Pas de Modification 4.0 International. 


\title{
Lost in Lost : \\ Entre quotidien anodin et déstabilisation fantastique, entre réalité alternative et fiction collective

\begin{abstract}
Cette communication prolongera les réflexions sur les procédés narratifs de Lost, qui brouillent les limites entre le « réel » et le fantastique. Il s'agira cette fois-ci d'analyser la stratégie narrative qu'offre la Saison 6 à travers le procédé original du "flash-sideways " (ou « flash-ahead »). Les « flash-sideways » de Lost mettent au défi la compréhension des spectateurs, brouillant tout repère narratif et temporel et incitant à la construction d'une véritable théorie du récit. Les «flash-sideways » oscillent jusqu'au bout entre la construction d'une réalité temporelle alternative (qui fonctionne comme un palimpseste réflexif-la série se réécrivant elle-même) et une fiction collective créée par les personnages eux-mêmes-rêve (ou œuvre d'art) post-mortem où chaque vie devient une mémoire qui ressurgit par bribes, révélant aux protagonistes ce qu'ils ont été, dans une déconstruction (toujours émotionnelle) de la fiction et un rappel de ce que les spectateurs ont eux-mêmes "vécu » depuis six saisons.
\end{abstract}

$\mathrm{C}$ et article propose une analyse de la stratégie narrative qu'offre la série américaine Lost (ABC, 2004-10) pour égarer les spectateurs dans des limbes entre fiction et réalité. Il s'agira de commenter les ouvertures des saisons 2 (épisode "Man of Science, Man of Faith »), 3 (épisode "A Tale of Two Cities ») et 5 (épisode « Because You Left »), ainsi que le procédé original du «flash-sideways » offert par la sixième et dernière saison. Les ouvertures et les "flashsideways » de Lost brouillent tout repère narratif et temporel, mettent au défi la compréhension de l'histoire par les spectateurs, et incitent à l'élaboration d'une véritable théorie du récit ainsi qu'à une réflexion sur la création même de la fiction télévisuelle.

Lors de la saison 1 de Lost, un avion s'écrase sur une île du Pacifique. Quarante-huit passagers échappent de peu à la mort. Perdus dans une zone inconnue du globe, ils doivent rapidement affronter le caractère à la fois hostile et mystérieux de l'̂lle. En pleine jungle, ils découvrent notamment une trappe en verre et en métal, hermétiquement close, et vont tout faire pour essayer de l'ouvrir et découvrir ses secrets. La Saison 1 se termine avec l'explosion de la trappe à la dynamite. Suspense - il faudra attendre le début de la saison 2 pour apprendre ce qu'elle recèle. 
La Saison 2 s'ouvre... sur un œil qui s'ouvre, tout comme la Saison 1 avait commencé sur l'œil de Jack, s'éveillant dans la jungle à la suite du crash $^{1}$.

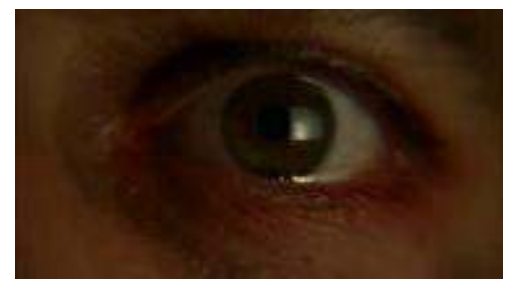

Le gros plan sur l'œil est paradoxal : il souligne l'importance de la vision, de la compréhension du monde autour de nous, tout en cachant l'environnement global au spectateur. Le plan montre qu'il cache: même si nous sommes en contact intime avec cet œil, nous ne savons rien du personnage à qui il appartient, ni où ce personnage se situe. Au début de cette séquence, certains spectateurs croyaient s'être trompés de chaîne tant on semblait s'éloigner de l'univers de Lost fait d'océan et de jungle. Certains pensaient cependant assister à une scène de flashback - un procédé narratif fortement utilisé lors de la Saison 1, où le passé des héros se trouvait peu à peu reconstitué devant nos yeux. Mais alors, de quel héros pouvait-il s'agir? Par son cadrage, la séquence garde l'identité du personnage totalement secrète.

La bande-son est étonnante: avec un bip lancinant qui ressemble au bruit d'un produit passant à la caisse d'un supermarché, une alarme réveille l'homme inconnu, qui se met aussitôt à taper quelques touches d'un ordinateur vieilli.
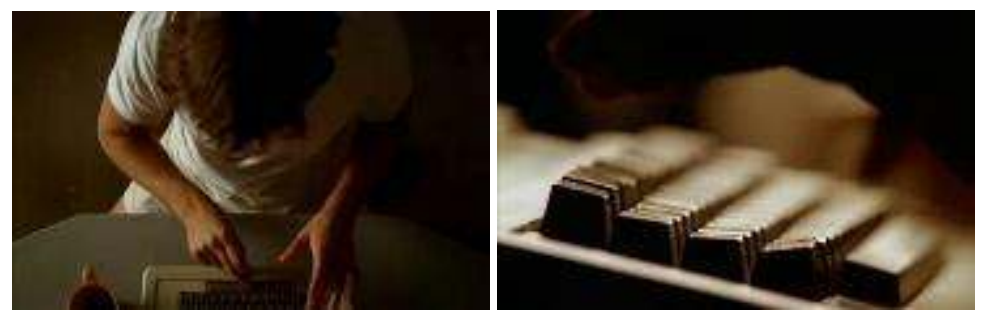

L'homme commence ensuite sa journée : choix d'un disque, vaisselle, sport, douche, mise en route de la machine à laver et du mixer (clin d'œil à la remise en route de la série ?), petit déjeuner...

${ }^{1}$ L'ouverture de la Saison 2 peut être visionnée ici (lien consulté en mai 2011) http://www.youtube.com/watch?v=g_5ZQWoBCFU 

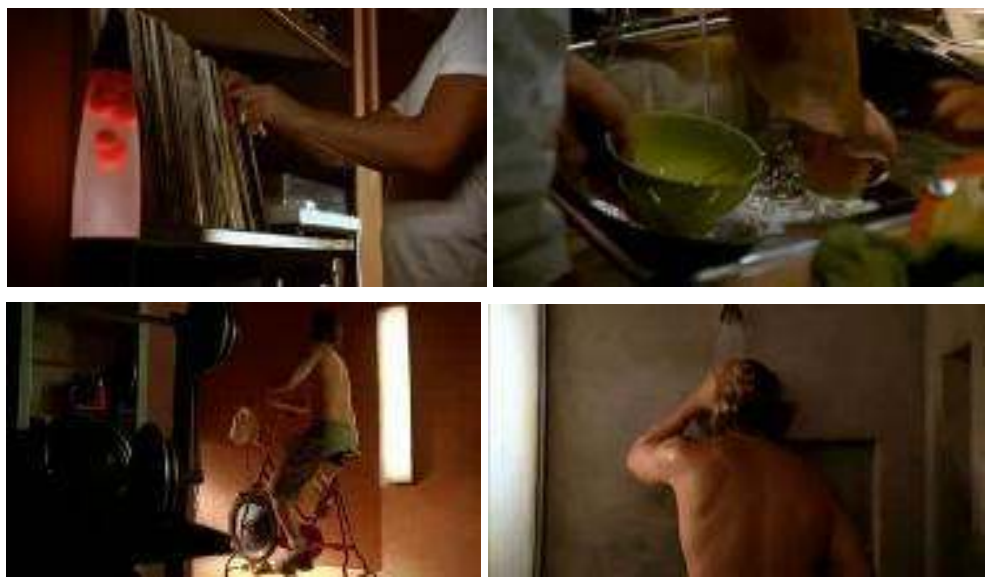

Des tâches quotidiennes presque normales et anodines, si l'on passe outre l'injection étrange d'un produit dans le bras. Mais peut-être s'agit-il d'un sportif dopé ou hyper-vitaminé ? Un élément, surtout, nous frappe: l'importance donnée au disque vinyle et au morceau musical Make Your Own Kind of Music des Mamas \& Papas (1969). La mélodie accompagne toute la première partie de la séquence, et lorsque nous l'écoutons rétrospectivement, commente la situation du personnage de Desmond (qui n'est encore qu'un inconnu à ce stade), à la fois isolé et obligé de s'inventer un mode de vie bien à lui. Le vinyle et la musique donnent également des indices précieux sur l'époque à laquelle l'appartement a été construit. Cependant, pendant toute la première partie de la séquence, la musique nous dupe. Lorsque Desmond met le tourne-disque en route, nous pensons avoir droit à une musique diégétique, censée durer autant de temps pour le personnage dans l'histoire que pour les spectateurs. Or, dans le montage séquentiel qui suit, nous montrant Desmond vaquant à toutes sortes d'activités avec des ellipses, cette équivalence temporelle ne peut avoir lieu: la musique est, en fait, subrepticement devenue extradiégétique avant de redevenir diégétique lorsque le choc d'une étrange explosion fait arrêter le tourne-disque. Cette oscillation musicale est à l'image d'une série qui joue sans cesse avec les repères du temps et de l'espace («There is no now here » est d'ailleurs l'une des dernières répliques de la série), mais aussi avec ce qui semble être une réalité quotidienne et anodine mais qui recèle finalement bien des mystères et se révèle construction fictive. Ce début de séquence peut aussi se lire comme une métaphore du processus de création filmique : écriture du scénario sur un ordinateur (Desmond tapant les touches), production exécutive (Desmond pressant la touche «execute»), action (tâches 
ménagères, mise en route du matériel), post-production (ajout de la musique).
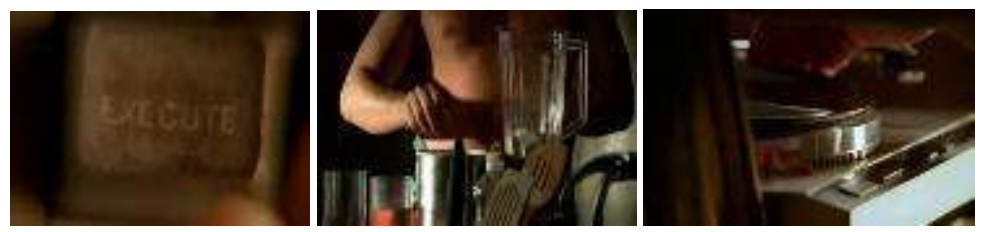

À la fin de cette séquence, le changement de ton est conséquent : d'un milieu confortable, presque familier, nous passons à un environnement de guérilla: nouveau costume, musique extradiégétique atonale, qui tranche avec la mélodie joyeuse et insouciante de Make Your Own Kind of Music. La séquence met à nouveau l'accent sur la vision et le regard: par le jeu de miroirs et de longue-vue, Desmond tente de découvrir ce qui a causé l'explosion et perturbé sa matinée : c'est là que les spectateurs découvrent l'inversion magistrale de cette ouverture dans tous les sens du terme - ouverture de la trappe et de la saison. Nous nous attendions à voir nos héros découvrir l'intérieur de la trappe ; mais nous y étions déjà et c'est en fait depuis l'intérieur que nous allons découvrir Jack et Locke, penchés vers nous pour essayer de percer le mystère. Le réalisateur Jack Bender décrit ainsi cette stratégie d'ouverture : "We wanted to show what's inside before we even know it is inside ${ }^{2}$ ». Les survivants voyeurs sont devenus ceux qui sont scrutés - par Desmond et par nous ; mais nous devenons également l'objet du regard de Locke et Jack, comme si les héros étaient finalement désireux de percer à jour le mystère même de leur construction fictionnelle et nous découvrir de l'autre côté de l'écran.

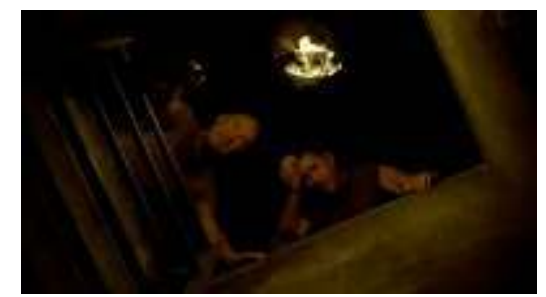

La Saison 2 nous fera découvrir les Autres - les habitants de l'île arrivés bien avant les survivants; mais elle nous emmènera très souvent dans la trappe, qui va devenir le QG des survivants - à la fois source de réconfort mais aussi d'angoisse. Desmond leur a expliqué

${ }^{2}$ Voir le commentaire du réalisateur en bonus du DVD. 
qu'il fallait, toutes les 108 minutes, entrer un code dans l'ordinateur 4815162342 - pour remettre à zéro un compteur et éviter un incident électromagnétique qui serait fatal à l'île - et peut-être même au monde. Voici donc l'explication de l'activité de Desmond au saut du lit dans la séquence d'ouverture. Il s'agit aussi d'une réflexion plus profonde sur la croyance dans le récit - les héros se divisent entre ceux qui croient à la nécessité de cette mission et ceux qui voient en cette activité une expérience fantaisiste, une manipulation extérieure à dédaigner. Les personnages viennent refléter alors les interrogations des spectateurs eux-mêmes qui se sont souvent divisés sur les forums internet, entre ceux qui croyaient en une trame narrative contrôlée par les créateurs dans ses moindres détails et ceux qui pensaient que les scénaristes naviguaient à vue et ne contrôlaient que les spectateurs dupés.

Le compteur est également la littéralisation du processus narratif à l'œuvre dans Lost : la série évite la linéarité, jouant sur la réécriture, la répétition en variation, la reprise dans l'instabilité, se passant souvent d'explications pour se concentrer sur l'efficacité des situations dans l'instant ; mais c'est aussi aux spectateurs de remettre leurs propres compteurs à zéro en réévaluant constamment les situations à l'aune de nouveaux indices.

Ce processus est porté à son apogée dans la séquence d'ouverture de la Saison $3^{3}$. Répétition : ouverture de l'œil ; variation : il s'agit d'une femme, et elle est immédiatement identifiée comme Juliet, l'une des Autres que l'on a aperçue à la fin de la Saison 2.
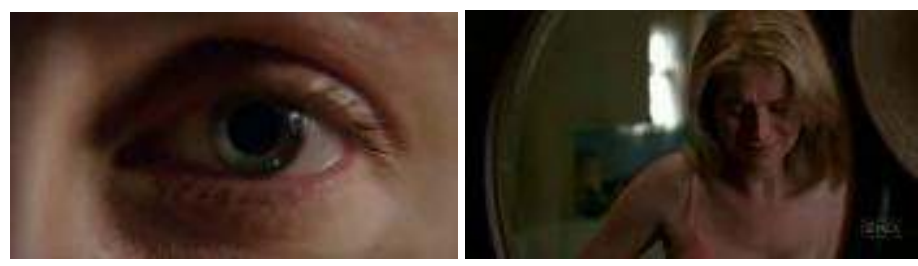

Répétition : elle choisit un disque à écouter ; variation : il s'agit d'un CD et non plus d'un vinyle - un autre indice sur la date de construction $\mathrm{du}$ lieu. Une chanson des années 60 à nouveau, mais un autre titre, Downtown de Petula Clark.

${ }^{3}$ L'ouverture de la Saison 3 peut être visionnée ici (lien consulté en mai 2011) : http://www.youtube.com/watch?v=8IEQLJmubF8 

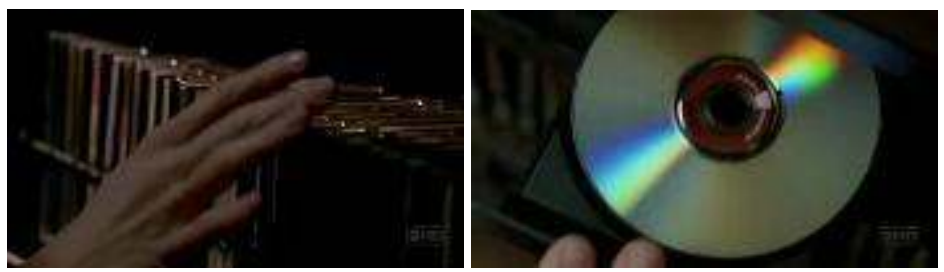

Variation sur les tâches ménagères: cette fois-ci il s'agit du réagencement des meubles car des invités sont attendus ; variation sur l'alarme : cette fois-ci c'est celle du four qui se déclenche; pâtisserie ratée, petite brûlure - événements anodins dans la vie d'une femme qui essaye de profiter du quotidien mais n'arrive pas à (se) cacher son désespoir.
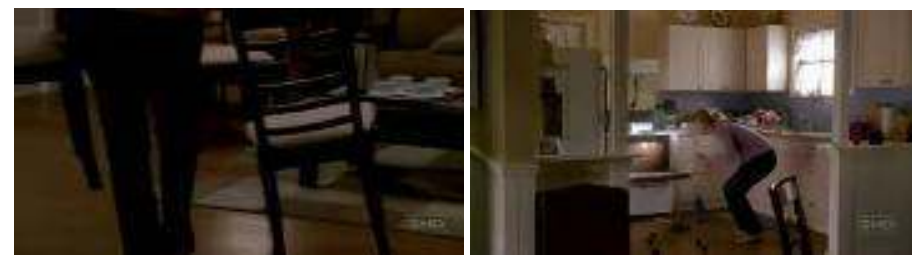

L'on se croirait dans une paisible banlieue d'une ville américaine où les voisins s'entraident ou s'invitent les uns chez les autres pour parler de livres ou de cuisine. La Saison 3 de Lost brouille encore une fois les codes attendus et commence comme Desperate Housewives.
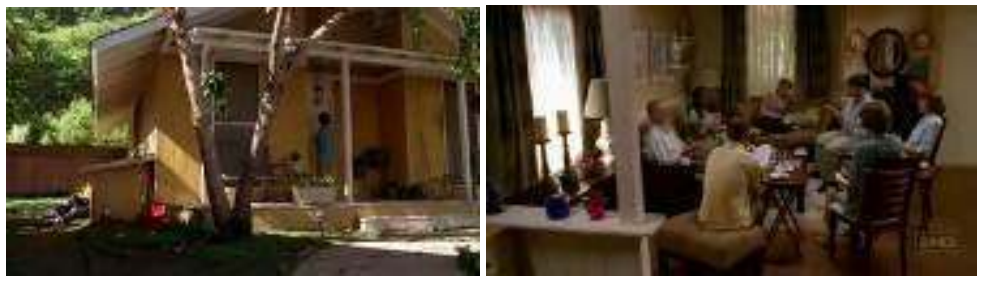

Les spectateurs peuvent à nouveau s'imaginer qu'il s'agit d'un flashback révélant le passé de Juliet avant sa venue sur l'île. La discussion du petit groupe de voisins autour d'un livre, qui serait « trop populaire », " pétri de fatras religieux » et de « science-fiction », donne à Lost l'occasion de parler d'elle-même et de l'opposition souvent artificielle entre la culture low brow et high brow, mais la série introduit aussi la question du libre-arbitre sur laquelle les saisons fondées sur le voyage dans le temps s'interrogeront constamment. Le livre qui fait débat s'avère être Carrie de Stephen King, écrivain connu 
pour introduire des éléments fantastiques dans la routine du quotidien. Or, c'est ce qui va se produire encore une fois dans la seconde partie de cette séquence d'ouverture, lorsque survient une secousse que l'on prend tout d'abord pour un tremblement de terre. Les personnages sortent des maisons pour comprendre ce qui vient de se passer. Survient alors le choc pour les spectateurs de voir Ben sortir de l'une de ces petites maisons banlieusardes.
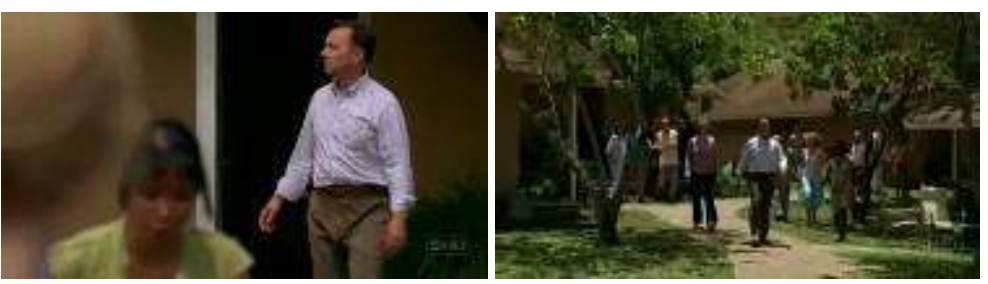

Ben, l'un des Autres de l'île, est devenu un personnage récurrent de la Saison 2, l'ennemi par excellence des héros survivants. Que fait-il alors dans le passé de Juliet ? Serions-nous alors dans le présent ? Et sur l'île ? Or nous n'avions encore jamais vu les Autres vivre autrement que dans des caches souterraines ou des huttes en bois. La musique souligne le malaise et la perte de repère des spectateurs. Ce n'est qu'en voyant l'avion se disloquer dans le ciel que nous comprenons où et quand nous nous situons dans l'histoire : il s'agit bien d'un flashback, mais qui nous ramène au début de la série vu à travers le regard des Autres. Une autre répétition dans la variation. Une autre remise à zéro du compteur.
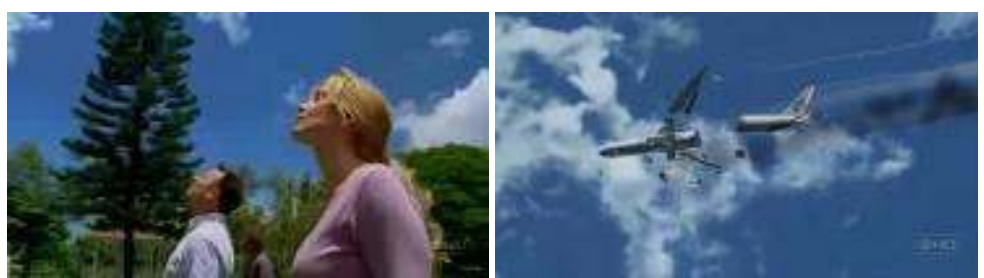

Nous comprenons comment les personnages de Goodwin et Ethan ont été initialement envoyés parmi les survivants pour les espionner et nous reconstituons une partie du puzzle. Les Autres vivent donc de manière confortable et moderne sur l'île, que nous pensions être un lieu uniquement sauvage et hostile. La civilisation serait-elle donc à portée de main pour les survivants ? Où sommes-nous ? L'île est-elle proche d'un continent? Quatre changements d'échelle successifs complètent alors la nouvelle donne et recontextualisent à nouveau le 
récit : les Autres vivent dans une clairière en plein cœur de l'île, perdu au milieu de nulle part.
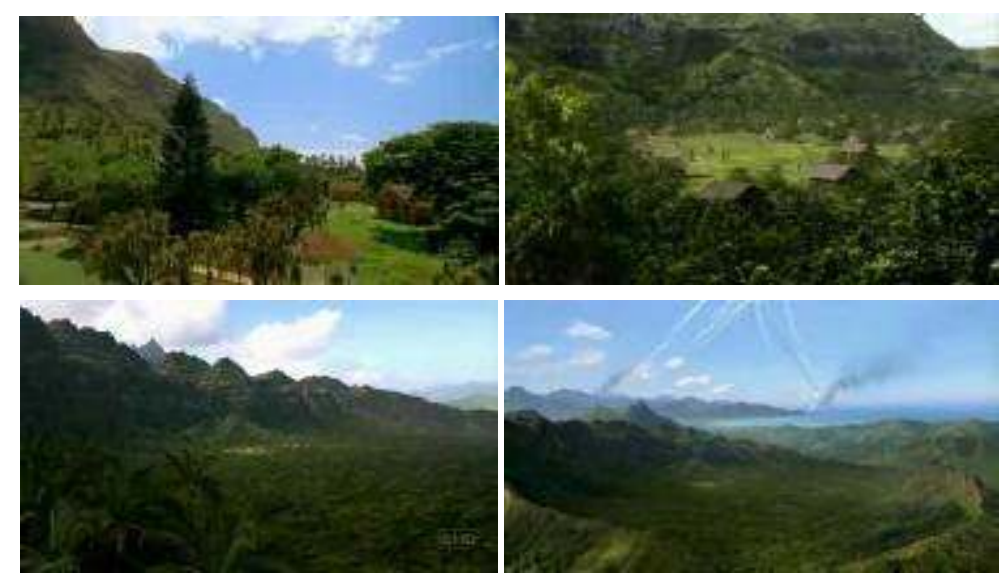

D'autres questions surgissent alors : comment se sont-ils installés sur l'île et dans quel but? Dans un bonus du DVD de la Saison 3, l'une des productrices a précisé que le changement d'échelle fonctionnait comme un épisode de la série La Quatrième dimension, où la caméra avait l'habitude de prendre de la distance et révélait que l'histoire se déroulait finalement dans une goutte d'eau au fond d'un verre. Une réalité en cache une autre. Dans Lost, le changement d'échelle nous révèle que les maisons ne sont pas dans la banlieue de Los Angeles mais perdues au milieu de la jungle et de l'océan ; il contribue ainsi à montrer ces maisons comme un décor fabriqué - comme si les Autres avaient construit un véritable décor de cinéma pour y vivre en personnages. Cette tension entre " réalité » et fiction, vie quotidienne et illusion, se retrouve notamment lors du deuxième épisode de la Saison 3. Dans l'épisode « The Glass Ballerina », Ben retient Jack, Kate et Sawyer prisonniers ${ }^{4}$. Il explique à Jack que s'il accepte de coopérer, il pourra ensuite rentrer chez lui facilement. Pour lui prouver qu'il est en contact permanent avec le monde extérieur, Ben l'informe de ce qui s'est passé aux Etats-Unis depuis le crash de l'avion. Des faits réels (la réélection de Bush, la mort de Christopher Reeve, la victoire des Red Socks au championnat de baseball) font intrusion dans le monde de la fiction. Le plan où Jack découvre, à travers la vitre de sa cellule, l'événement sportif réel sur un écran de télévision, avec le reflet de l'émission sur son visage, est symptomatique: les personnages de

${ }_{4}$ Cette séquence de la Saison 3 peut être visionnée ici (lien consulté en mai 2011) : http://www.youtube.com/watch?v=jAhAWaZUfdw 
fiction sont présentés comme faisant partie de notre monde - ce qui les rend plus "vrais ", mais révèle aussi notre "réalité » comme une construction médiatique.

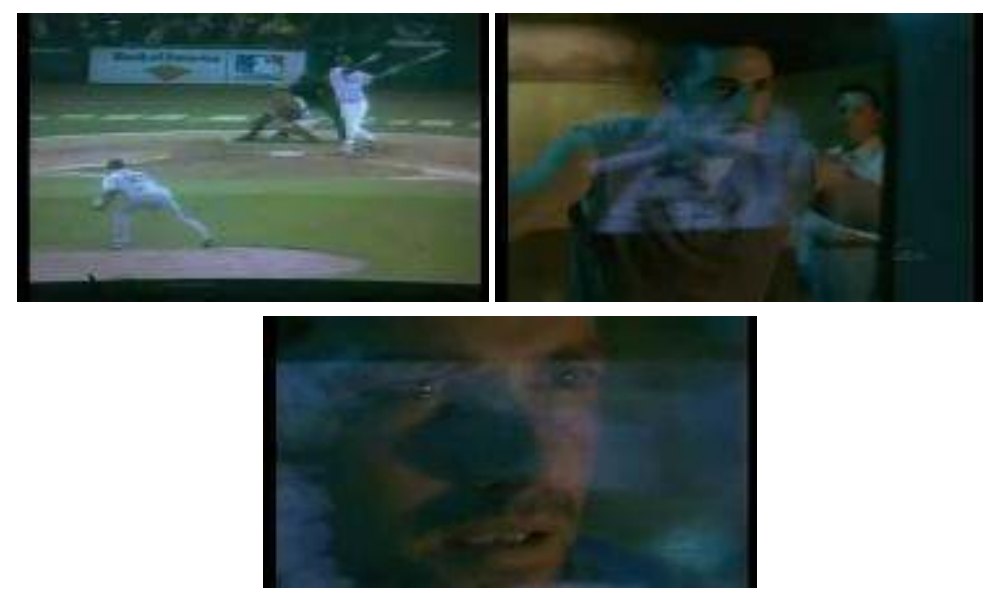

Dans cette séquence, la télévision est censée fonctionner comme une preuve donnant à voir le vrai. Mais paradoxalement, et de manière fort métafilmique, elle finit par révéler la fiction. Lorsque Ben dit, «That's home, Jack. Right there, on the other side of that glass ", il révèle notre propre existence de spectateurs derrière l'écran de télévision. Cette réflexion sur la construction filmique se poursuit dans la séquence d'ouverture de la Saison $5^{5}$.

Variation sur le même thème : réveil d'un personnage ; réveillematin rappelant le compteur de la Saison 2 et jouant sur le numéro du vol accidenté, Oceanic 815; cadrage ne permettant pas d'identifier immédiatement le personnage masculin, mise en marche d'un tournedisque, tâches quotidiennes (cette fois-ci, c'est d'un bébé qu'il faut s'occuper), douche, rasage...
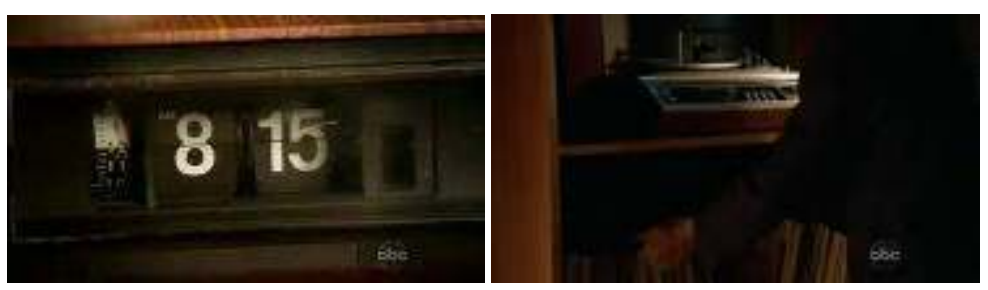

${ }^{5}$ L'ouverture de la Saison 5 peut être visionnée ici (lien consulté en mai 2011) : http://www.youtube.com/watch?v=qIJBXFN37bM 

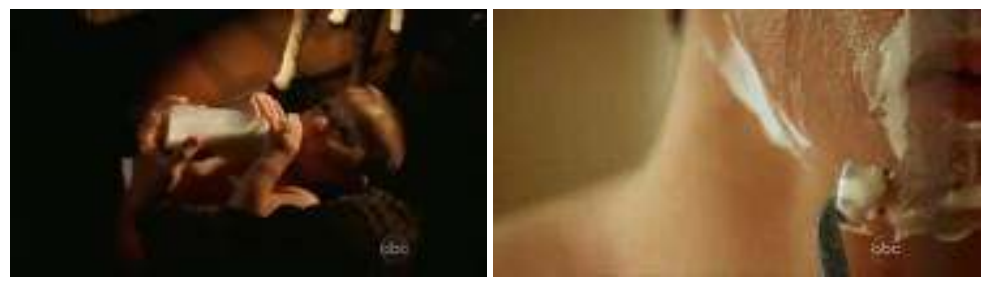

A travers la figure littéralisée du disque rayée, la série commence d'ailleurs à se moquer d'elle-même et des échos qu'elle met en place. La bande sonore renvoient les mêmes paroles, " if you can't make a record " à plusieurs reprises, soulignant cette notion de disque rayé mais aussi celle d'enregistrement - ce que le personnage s'apprête justement à faire.
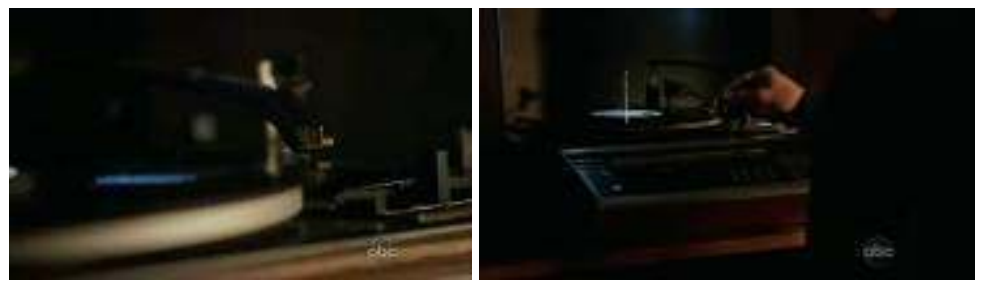

On découvre qu'il s'agit du Professeur Pierre Chang, qui a occupé les petites maisons de l'île avant les Autres, au temps de l'expérience scientifique menée par la Dharma Initiative. Au cours des saisons précédentes, nous n'avions connu Chang qu'à travers des films super-8 des années 70 où il expliquait le fonctionnement des stations Dharma (la fameuse trappe était l'une d'entre elles). Nous comprenons alors que cette séquence d'ouverture nous a propulsés trente ans en arrière lors du tournage de ces petits films. De manière métafilmique, la série ironise sur son propre rythme de production ( « Let's go, I don't have all day » dit Chang) et donne peut-être un indice capital sur sa stratégie de création narrative («I don’t need a script», poursuit-il) ou, au contraire, met en avant à quel point elle est scénarisée par rapport à ces petits films de présentation.
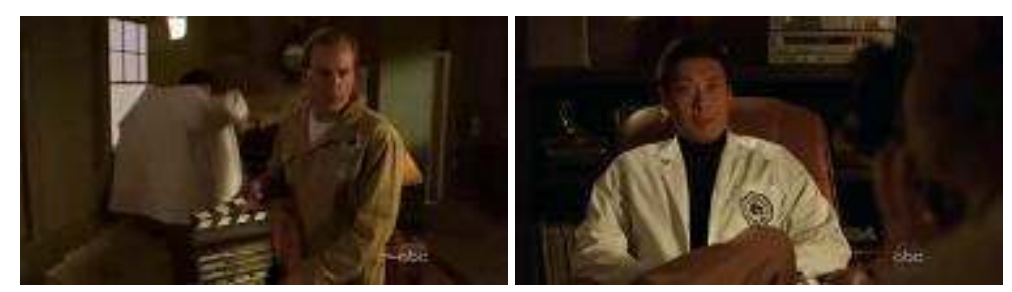
La routine du tournage est interrompue pour laisser place à nouveau au fantastique. Le changement qui nous amène à tout repenser a lieu mais cette fois-ci ce sont nos repères temporels, et non géographiques, qui sont mis à mal. Ironiquement, alors que nous venons juste d'entendre un ouvrier dire que le voyage dans le temps est impossible (« Time travel. How stupid does that guy think we are? »), nous apercevons Daniel Faraday, un personnage que nous savons appartenir aux années 2000 et comprenons donc qu'un voyage dans le temps a déjà eu lieu.

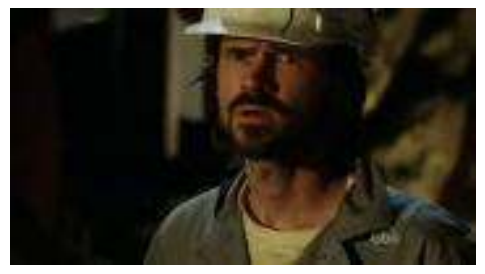

Cependant, la série se distingue d'emblée des récits classiques sur le voyage dans le temps où le cours de l'histoire se trouve transformé. Avec le dialogue suivant, " - We're going to go back and kill Hitler? » « - Don't be absurd. », Nous sommes prévenus dès le début : le cours du temps ne peut être changé - ce qui est arrivé est arrivé. De manière révélatrice, la série promet une manipulation temporelle grâce aux propriétés électromagnétiques de l'île (« it will allow us to manipulate time »). Lost va nous inciter à croire en d'étranges constructions du récit, comme si l'énergie de l'île était la métaphore même de la création narrative.

La force de la série Lost ne réside certainement pas dans sa capacité à répondre aux questions qu'elle a posées tout au long des saisons, mais dans la liberté qu'elle laisse aux spectateurs de faire des liens, de repérer les échos, de jouer avec les axes de l'espace et du temps, et de, finalement, composer leur propre théorie narrative, leur propre réalité de la fiction - tout comme les personnages composeront leur propre monde alternatif dans les « flash-sideways » de la Saison 6. Lost contient, en effet, sa propre problématique ou tension narrative : ces « flash-sideways » oscillent jusqu'au bout entre réalité alternative offerte par les scénaristes et fiction collective créée par les personnages eux-mêmes. Cette oscillation témoigne d'une déconstruction paradoxalement toujours émotionnelle de la fiction, qui joue à la fois sur les émotions et les souvenirs des personnages et sur ceux des spectateurs. 
Dans le Pilote de la série, diffusé en 2004, Jack, survivant du vol 815, se souvient des moments dans l'avion qui ont précédé l'accident. Le flash-back s'ouvre sur le ciel vu par le hublot ${ }^{6}$. Nous voyons Jack se plaindre de sa boisson (qui n'est pas assez forte selon lui), se faire offrir deux mignonettes par l'hôtesse de l'air, puis entamer une conversation avec sa voisine, Rose, qui fera elle aussi partie des survivants.
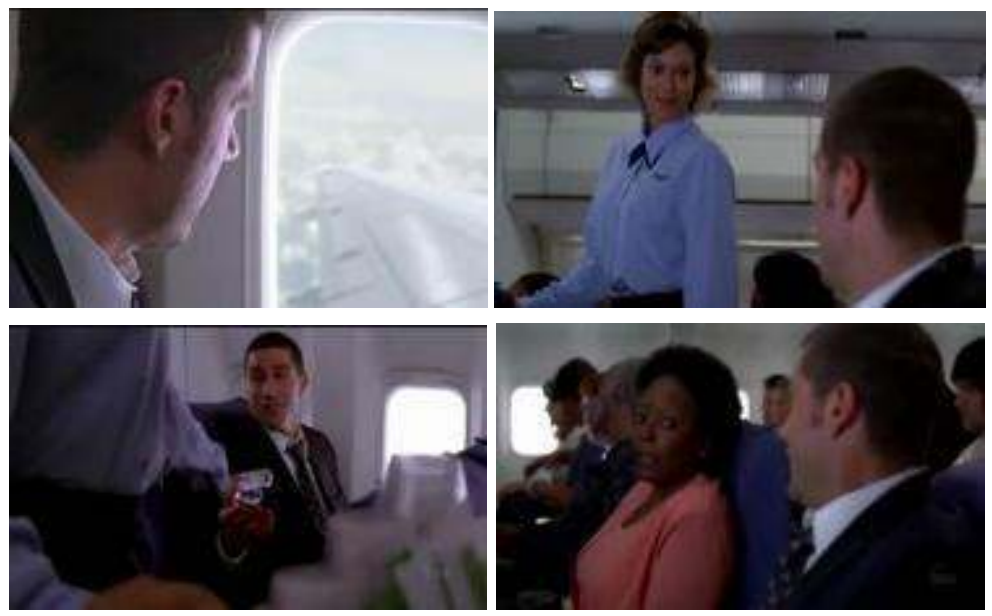

Lorsque l'avion subit une première série de secousses, c'est Jack qui rassure Rose : «It's normal ». Mais au moment où il s'apprête à finir sa phrase « Don't worry, it's gonna be all... », les secousses s'intensifient, les masques à gaz tombent et l'accident devient inévitable. Le crash de l'avion précipitera les personnages sur l'île, qu'ils ne quitteront plus pendant des mois pour certains, pendant plusieurs années pour d'autres.
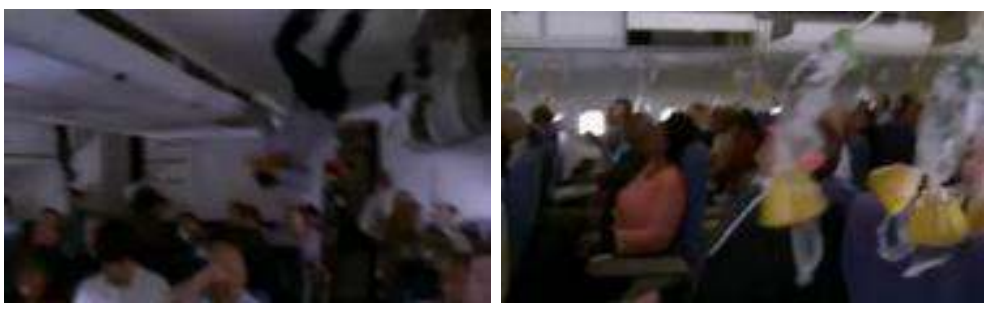

${ }^{6}$ Ce flashback (Saison 1, Pilote première partie) peut être visionné ici (lien consulté en mai 2011) : http://www.youtube.com/watch?v=zeZrAh5nz2k 
Lors de la Saison 5 des dérèglements électromagnétiques précipitent les personnages dans le temps. Ils sont projetés trente ans dans le passé, dans les années 70, alors que la Dharma Initiative mène des expériences scientifiques sur les propriétés de l'île. Pour essayer de changer le cours du temps, ils vont alors tenter de déclencher une explosion nucléaire en utilisant une bombe laissée par l'armée américaine après la guerre du Pacifique. Les personnages fondent leurs espoirs sur la théorie suivante : si l'île explose dans les années 70, elle n'existera plus en 2004; ses effets électromagnétiques ne pourront plus affecter les avions qui passeront au-dessus d'elle et le crash du vol 815 n'aura donc pas lieu. Lors d'un incident électromagnétique majeur, la bombe est jetée dans le puits d'une des stations Dharma en construction, mais elle n'explose pas. Juliet, tombée dans le puits par accident et s'estimant au seuil de la mort, déclenche le détonateur. La Saison 5 se termine dans la lumière aveuglante de l'explosion. Le cours du temps va-t-il alors être changé ? Les règles du jeu temporelles sans cesse rappelées pendant cette cinquième saison - à savoir "What happened happened » - vont-elles enfin être transgressées ${ }^{7}$ ?

La Saison 6, diffusée en 2010, s'ouvre sur un rappel de cette explosion blanche, qu'elle prolonge visuellement par les nuages perçus à travers le hublot d'un avion ${ }^{8}$. La première séquence est donc esthétiquement présentée comme la conséquence directe de l'explosion nucléaire. Revoici Jack dans le vol 815 : tout ressemble à s'y méprendre à ce que nous avons vécu lors de la diffusion du Pilote en 2004, à quelques différences près. Comme les ouvertures des Saisons 2, 3 et 5 , cette séquence joue sur les répétitions en variations, sources de fantastique au cœur même du quotidien. Les spectateurs qui ont suivi la série depuis le début sont alors confrontés à du déjà-vu, à du déjàvécu : pour eux, il s'agit de la deuxième occurrence de la séquence. Cependant, pour Jack, tout semble nouveau : il a certes d'abord le regard d'un homme qui s'éveille d'un long rêve, reflétant ainsi l'incrédulité des spectateurs devant ce retour au point de départ, cette nouvelle remise à zéro des compteurs, mais il parait ensuite vivre tous les événements pour la première fois. Ce décalage entre l’ignorance du personnage et la connaissance des spectateurs n'est pas pour autant source d'une simple ironie dramatique car, si nous connaissons la situation, nous ignorons si tout se déroulera la deuxième fois sur le modèle de la première. L'horizon d'attente des spectateurs est passé en mode binaire : soit tout se reproduit à l'identique, l'accident a lieu et le cours du temps n'a pas été affecté ; soit tout ne se déroule pas à l'identique et nous pourrions alors en conclure que l'explosion de la

\footnotetext{
7 Pour une réflexion sur le voyage dans le temps dans Lost, voir Kristine Larsen, « The Art of World-making: Lost and Time Travel ", Lost Online Studies 2.1, http://loststudies.com/2.1/lost time travel.html. Consulté en mai 2010.

${ }^{8}$ L'ouverture de la Saison 6 peut être visionnée ici (lien consulté en mai 2011) :

http://www.youtube.com/watch?v=oQZcMQWTAZU
} 
bombe a bel et bien affecté le présent ou, tout du moins, ouvert un embranchement temporel dans lequel le vol 815 ne s'écrase pas - une réalité alternative ou «flash-sideways », qui viendrait matérialiser «l'autre vie » évoquée par Desmond dans sa phrase-mantra, «See you in another life, brother ».

Les spectateurs sont alors encouragés à se transformer en détectives, guettant le moindre indice pour valider l'une ou l'autre des théories narratives. Cette interaction avec la série, qui demande un regard particulièrement attentif, quasi scrutateur, a conduit le critique Jason Mittell à penser le concept de «forensic fandom » 9 . Ce désir qu'ont les spectateurs "légistes » à voir et revoir les séquences pour mieux en percer les mystères s'apparentent à ce que vivent les personnages devant les films amateur de la Dharma Initiative : John Locke, immédiatement après avoir regardé les instructions en Super-8 de la station du Cygne, réagit ainsi : "We're going to need to watch that again ». Lost joue sur ce que Peter Brooks a appelé l'anticipation de la rétrospection: nous voulons (et nous croyons) pouvoir nous retourner sur l'œuvre en comprenant comment chaque élément a construit le sens global ${ }^{10}$.

Lors des premières secousses de l'avion, nous pouvons penser que tout va se répéter, mais d'autres indices nous laissent supposer que tout sera différent : Jack, aux cheveux légèrement plus longs, n'est plus assis à la même place dans l'avion ; l'hôtesse de l'air donne à Jack non plus deux mignonettes, mais une seule ; Rose porte une autre tenue et cette fois-ci c'est elle qui rassure Jack après la première turbulence : «It's normal », lui dit-elle, dans une inversion des rôles. Les différences dans le cadrage et le montage frappent particulièrement : la volonté est claire de ne pas réciter plan par plan la séquence d’origine mais d'introduire des variations en s'appuyant sur un sentiment intense de familiarité.

9 Jason Mittell, «Lost in a Great Story: Evaluation in Narrative Television (and Television Studies) ", in Reading Lost: Perspectives on a Hit Television Show, éd. Roberta Pearson, London, I.B. Tauris, 2009, p. 128-9 [119-38].

10 "the anticipation of retrospection ", Peter Brooks, "Reading for the Plot: Design and Intention in Narrative ", New York, Knopf, 1984, p. 22, cité par Ivan Askwith, "'Do You Even Know Where This Is Going?': Lost's Viewers and Narrative Premeditation ", in Reading Lost: Perspectives on a Hit Television Show, dir. Roberta Pearson, London, I.B. Tauris, 2009, p. 169 [159-80]. 

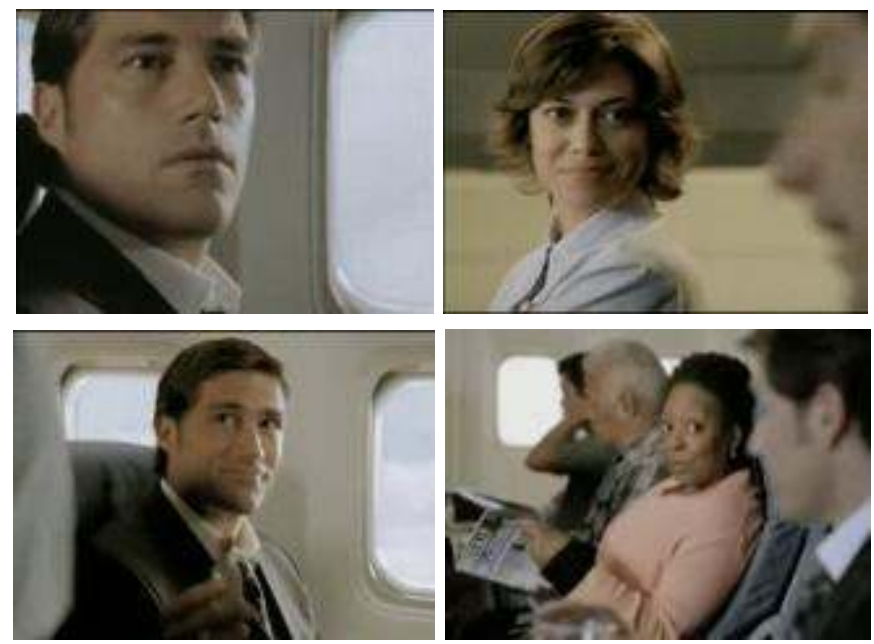

Lorsque la seconde turbulence ne donne pas lieu à un crash, Rose et Jack ont un échange bref mais lourd de sens quant au statut narratif de toutes les séquences de flash-sideways :

Rose: - You can let go. It's OK, you can let go.

JACK: - It looks like we made it.

RosE: - We sure did.

Tous les «flash-sideways » qui ponctuent la Saison 6 semblent révéler ce qu'aurait été la vie des personnages si l'île n'avait pas existé, comme si la série se mettait à fonctionner sur le mode du palimpseste réflexif et commençait à se réécrire elle-même. Ce dialogue entre Rose et Jack lors du premier épisode de la Saison 6 donne pourtant déjà la clé du mystère : la réalité alternative n'est qu'une fiction, dans tous les sens du terme.

S'il nous semble pour l'instant que l'explosion nucléaire a ouvert une ligne temporelle parallèle en 2004, les personnages qui ont fait exploser la bombe dans le passé sont, eux, toujours dans la ligne temporelle d'origine. Miraculeusement, Juliet a survécu à l'explosion pour mourir quelques instants plus tard dans les bras de Sawyer, l'homme avec qui elle vient de vivre trois ans dans le passé de l'île. La séquence de la mort de Juliet, qui intervient dans le premier épisode de la Saison 6, donne encore des indices sur le statut narratif des « flashsideways » et fonctionne comme un écho au dernier épisode de la 
série $^{11}$. La première réaction de Juliet est de constater que l'explosion de la bombe ne les a pas tous propulsé dans un monde où le crash n'a jamais eu lieu : "It didn't work ». Elle semble ne pas avoir encore conscience de la ligne temporelle parallèle ; mais juste avant de mourir, elle semble s'adresser à quelqu'un qui n'est pas Sawyer («We could have coffee sometimes. We could go Dutch ${ }^{12} »$ ) comme si elle était entrée en contact avec un autre monde. Ce qu'elle tente de dire à Sawyer dans un dernier soupir est peut-être une information cruciale sur ce point. Une fois Juliet enterrée, Miles, personnage médium qui arrive à entendre les morts, parvient à capter le message qu'elle souhaitait tant communiquer : "It worked ». Les spectateurs peuvent alors en conclure que la bombe a littéralement généré un nouvel embranchement temporel.

Cependant, cette théorie s'effondre dans le tout dernier épisode de la série. Dans les "flash-sideways », les personnages vivent des retrouvailles et des chocs émotionnels qui réveillent leur mémoire : ce qu'ils ont vécu sur l'île leur revient à l'esprit. La ligne temporelle d'origine s'immisce, transpire, dans la nouvelle. La série joue alors sur l'idée d'un paradoxal traumatisme " heureux », du retour inespéré d'un refoulé enchanté, avant de nous dévoiler la nature de cette nouvelle ligne temporelle. Dans cette Saison 6, le cours du temps n'avait pas été changé mais prolongé : les «flash-sideways » sont des «flash-ahead », vie imaginée par les personnages après leur mort pour se retrouver avant de « lâcher prise » ( " let go ») et partir vers les horizons dont on ne revient jamais. La séquence où Juliet retrouve Sawyer dans le dernier épisode de la série repose sur de nombreux échos à la fois visuels et sonores ${ }^{13}$ : «It worked » fait, cette fois-ci, référence à un distributeur automatique ; les phrases «We could go and have coffee sometimes. We could go Dutch », qui apparaissaient incongrues avant la mort de Juliet, font ici partie du dialogue que l'on attend d'une relation qui (re)commence.
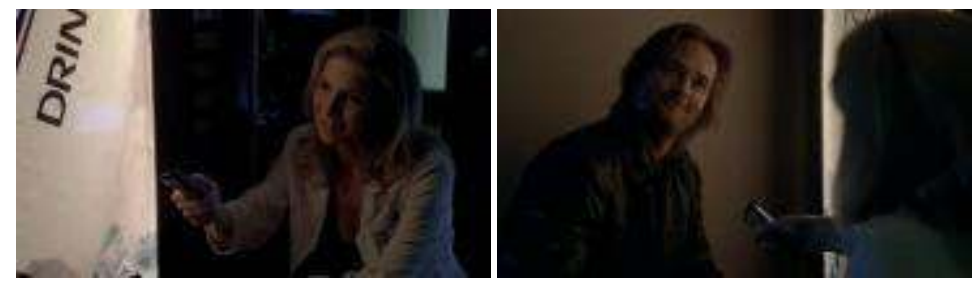

${ }^{11}$ La séquence de la mort de Juliet (Saison 6, épisode 1) peut être visionnée ici (lien consulté en mai 2011) : http://www.youtube.com/watch?v=TJA_E7jyiYg

${ }^{12}$ En anglais, « to go Dutch » signifie " partager l'addition ».

${ }^{13}$ La séquence des retrouvailles de Sawyer et Juliet (Saison 6, dernier épisode) peut être visionnée ici (lien consulté en mai 2011) :

http://www.youtube.com/watch?v=BQfo1JXnR_I 
Les souvenirs communs qui ressurgissent sous forme de flash-backs surexposés circulent entre les deux personnages, les relient tous les deux, tout en les connectant à leur passé. "We could go Dutch » est ainsi prononcé alors que la Juliet mourante est à l'écran. Juste avant de mourir, Juliet vivait déjà dans la réalité qu'ils allaient construire : de manière fort romantique, c'était donc bien à Sawyer, mais à un Sawyer d'un autre monde, que s'adressaient ces paroles à la fois prosaïques et prophétiques. La série abolit donc le temps et l'espace: les mêmes mots d'amour ressurgissent dans l'au-delà (« - Kiss me, James. You've got it, Blondie. »), il n'y a plus d'ici et maintenant, la mort n'est pas une fin ${ }^{14}$.
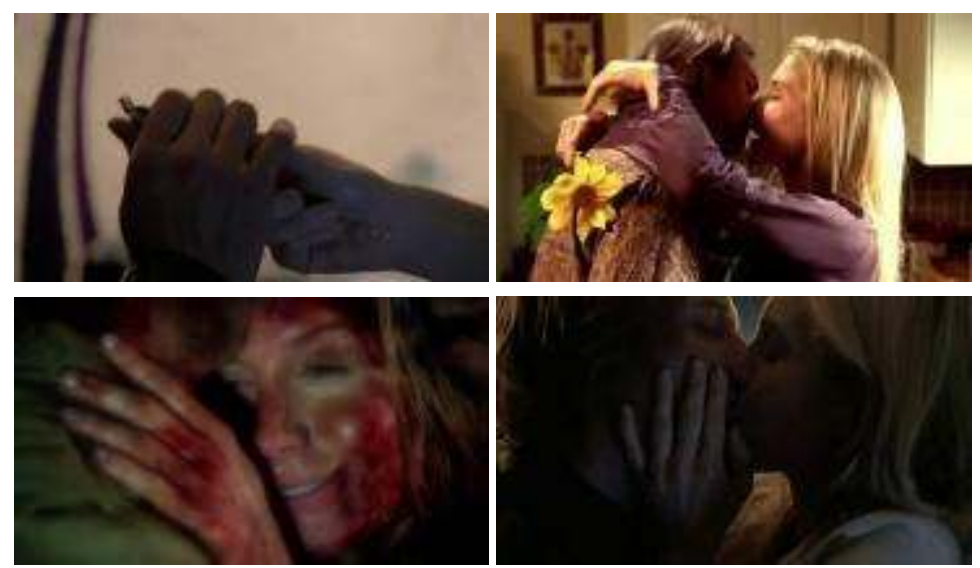

Dans le dernier épisode de la série, la figure du père, pourtant malmenée tout au long des saisons, est étrangement réhabilitée : Christian Shephard, le père de Jack, joue littéralement les bergers accompagnant les personnages vers l'acceptation de leur mort et ouvrant les portes de l'église pour laisser entrer une lumière que l'on suppose divine : cette ambiance spiritualo-religieuse où le respect pour la figure paternelle maltraitante est rétabli laisse pour le moins songeur, mais le moment où Jack retrouve son père décédé et prend

14 Voir l'étude de J.M. Berger sur le temps dans Lost, « past and present are not distinctly separated - and Lost's most compelling stories often stem from unusual transactions between the two timelines ", "Flashbacks, Memory and Non-Linear Time", Lost Online Studies 1.2, http://www.loststudies.com/1.2/memory-and-time.html. Consulté en février 2011 
conscience qu'il est à présent mort lui aussi est source d'une réflexion métafilmique sur les personnages de fiction ${ }^{15}$ :

JACK: - Are you real ?

CHRISTIAN: - I sure hope so. Yeah, I'm real. You're real. Everything that's ever happened to you is real. All those people in the church, they're all real too.

Tout au long de la Saison 6, cette obsession de Jack quant à sa « réalité » et à son statut fictif se traduit à l'image par des plans de miroir : Jack, Sawyer ou Locke se dévisagent, scrutent leur corps. L'image dédoublée, parfois fragmentée, vient refléter la double réalité du rôle et de l'acteur, ainsi qu'évoquer la figure d'identification qu'ils incarnent pour les spectateurs.
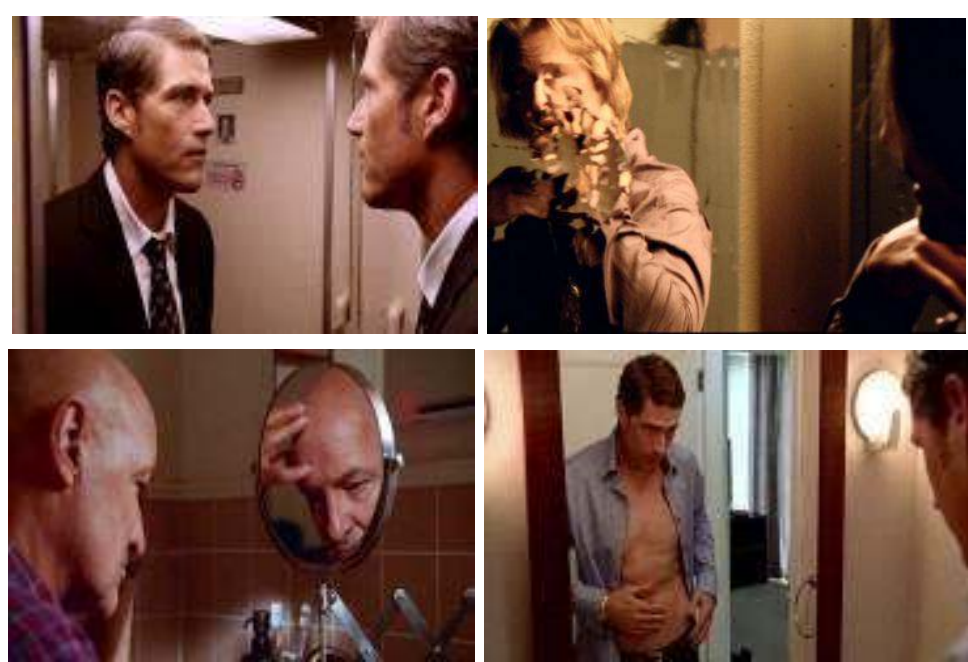

Michelle Lang estime que la série présente l'individualité comme une fiction, c'est-à-dire une construction culturelle, historique et sociale ${ }^{16}$. Il faudrait ajouter que l'inverse est vrai : dans une déconstruction paradoxale, le personnage de fiction est construit comme une réalité.

${ }^{15}$ La séquence où Jack retrouve son père (Saison 6, dernier épisode) peut être visionnée ici (lien consulté en mai 2011) : http://www.youtube.com/watch?v=Y8WPSiGBCAA

16 « Within its own fictional universe, the self is presented, in part, as a fictional construct. Certainly the characters are complex and realistic, but at the same time Lost has a certain new Historicism about it - identity is a social construction, mediated through the language of memory, and all truth is relative. ", Michelle Lang, "Lost: Poststructural Metanarrative or Postmodern Bildungsroman? ", Lost Online Studies 2.1

http://loststudies.com/2.1/postructural_metanarrative.html. Consulté en février 2010. 
Jack, héro qui s’interroge sur la réalité des autres personnages et de lui-même, nous renvoie à notre manière d'appréhender la fiction, que nous éprouvons souvent comme une véritable réalité alternative nous faisant vibrer quelquefois autant, parfois davantage, que nos vies ellesmêmes. Jack, confronté à l'idée de sa mort, met au jour son statut de personnage de fiction joué par un acteur et s'interroge lui-même, comme Hamlet face au suicide, sur son essence fictionnelle : « To be or not to be » (de manière fort révélatrice, Hamlet ne dit pas « To die or not to die »). Dans la première partie du tout dernier épisode de la série, les « flash-sideways » offrent des signes clairs de la mort de Jack, tel ce bref plan en forme de memento mori où Jack, dans son rôle de médecin, scrute la radio d'un crâne. Le plan superpose le crâne et le visage de Jack (vu en transparence), présente le personnage comme un spectre, tout le transformant en figure théâtrale par excellence, nouvel Hamlet tenant son célèbre accessoire.
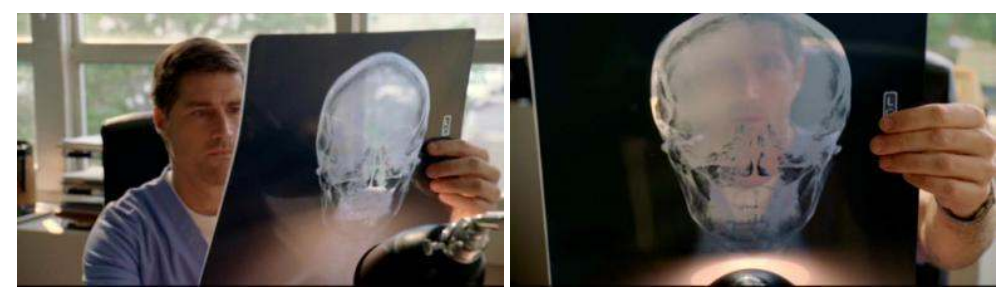

Christian confirme à Jack qu'il est bien réel (« You're real ») parce que, pour nous aussi, tout ce qui est arrivé est vrai ; toutes les émotions que nous avons ressenties en suivant Lost pendant six ans (ou quelques semaines, pour ceux qui ont visionné les DVDs de manière compulsive...) sont réelles. Dans une série feuilletonnante au long cours, le personnage de fiction acquiert une réalité d'autant plus grande que nous avons partagé sa vie pendant des années. Lorsque Christian dit à son fils, "You needed all of them and they needed you », il communique à nouveau l'idéologie du " vivre ensemble » de la série (qui n'a eu de cesse de prêcher un " Live together or die alone »), mais il dessine également les contours du contrat passé entre les œuvres de fiction et leurs spectateurs: nous avons besoin d'elles comme elles ont besoin de nous. Le «flash-ahead» a certes une fonction diégétique: il s'agit d'une fiction collective créée par les personnages eux-mêmes - à la fois rêve et ouvre d'art - leur permettant de se retrouver afin de se souvenir, de lâcher prise (« to remember and let go », nous dit Christian) et de partir vers l'inconnu :

JACK: - Kate... she said we were leaving.

CHRISTIAN: - Not leaving, no - moving on.

JACK: - Where are we going? 


\section{CHRISTIAN: - Let's go find out.}

Cependant, le «flash-ahead » a également une fonction extra/métadiégétique : il s'agit de préparer les spectateurs à la fin de la série, de les aider à faire un quasi-travail de deuil et de rétrospection: se souvenir en flash-back des moments marquants, se retourner sur le chemin parcouru, se rappeler ce que nous avons vécu depuis six ans, dans la fiction et hors de celle-ci, accepter qu'un récit se termine et passer à une autre fiction. Les personnages ne sont pas les seuls à devoir "passer à autre chose » : l'équipe du tournage va se séparer, chacun va se tourner vers des projets différents ; les spectateurs vont passer à d'autres séries, comme Flashforward (série au titre révélateur qu'on a, un temps, perçu comme la série succédant directement à Lost sur $\mathrm{ABC}$ en 2010) ou des fictions encore inconnues qu'il nous faudra découvrir («let's go find out »).

Dans Lost, chaque vie devient une mémoire qui ressurgit par bribes, révélant aux protagonistes ce qu'ils ont été et rappelant aux spectateurs ce qu'ils ont eux-mêmes ressenti. Le dernier épisode jette un véritable pont entre la diégèse et le monde des spectateurs comme l'attestent certains messages que les spectateurs ont postés sur des forums ou sur Youtube. En janvier 2011, un certain Bobbylight écrit ainsi :

i cried so much when LOST ended. along with the fact that the greatest show of our lifetime ended, but the moments you spent with your family watching lost. i watched it with my dad and brother and the best moments of my life where the ones watching lost with them. all the wtf moments we shared together nd lost pulled us closer together. so when christians talkiing its like hes talking straight to the viewer [sic] ${ }^{17}$.

L’idée de "faire lâcher prise " aux spectateurs était déjà présente au début de la Saison 6 lorsque Rose dit à Jack qu'il peut cesser de s'agripper à son siège ("You can let go now »), mais l'idée de création fictionnelle («This is a place that you all made together», dit Christian) apparaissait également au cours du même échange dans l'avion : «- It looks like we made it. - We sure did », où le jeu de mot entre " s'en sortir » et " fabriquer », la répétition du pronom «We » et la confirmation par un autre personnage insistent sur la notion de création collective. Cette idée de création collective inclut aussi les spectateurs qui, par leurs réactions sur les forums internet, ont contribué à stimuler et guider les scénaristes, ne serait-ce qu'en leur montrant ce à quoi les fans s'attendaient et les théories narratives que ces derniers construisaient au fur et à mesure ${ }^{18}$.

${ }_{17}$ Voir http://www.youtube.com/watch?v=Y8WPSiGBCAA

${ }_{18}$ Voir Askwith, op. cit., p. 159-80. 
À la fin de Lost, les personnages qui souhaitaient changer le cours du temps pour devenir les auteurs de leur vie n'en sont pas moins devenus les créateurs d'une fiction imbriquée, d'une mise en abyme du récit. Si les personnages ne détruisent jamais complètement l'illusion de la fiction, Michelle Lang remarque la tension qui existe dans Lost entre, d'une part, la protection de l'émotion et du " quatrième mur » et, d'autre part, la structure postmoderne de la série ${ }^{19}$. Cette tension existe, en fait, dans de nombreuses séries contemporaines : comme l'a souligné Jason Mittell, la complexité narrative des nouvelles séries et leurs expérimentations temporelles s'intègrent dans une esthétique opérationnelle ("operational aesthetics») qui pointe du doigt le processus d'élaboration du récit ${ }^{20}$. L'une des grandes caractéristiques des nouvelles séries est la présence de jeux scénaristiques incluant ce que l'on pourrait appeler des « plis hypothétiques ». Les séries jouent à "faire comme si..." et présentent en creux toutes sortes d'idées évoquées par les scénaristes en brainstorming, même si ces dernières n'intégreront pas la trame principale. Dans des séries comme Dexter, Desperate Housewives ou Six Feet Under, les brouillons et les ratures du script semblent prendre vie dans les séquences de rêve, de fantasmes, de coma, d'imagination.

L'originalité de Lost réside, en fait, dans la validation de ses propres plis hypothétiques : les "flash-sideways" ne sont pas de simples fantasmes que l'on pourrait considérer comme secondaires par rapport à une ligne narrative principale puisque leur statut oscille entre une réalité parallèle et un saut dans l'au-delà, "flash-forward » extrême (d'où le nom « flash-ahead » qui leur a été également attribué) dont la réalité est à nouveau réaffirmée dans les dernières séquences. A travers les "flash-sideways ", plis doublement fictifs mais validés comme réels à la fois par le récit et les personnages, la série inclut son propre remake, réfléchit à sa mort, à la mort, à ce que veut dire terminer un récit (et une histoire) qui a duré plusieurs années, et ce faisant tient un discours métafilmique qui révèle sa fictionnalité, tout en soulignant son impact bien réel sur nos vies et sur le monde.

\footnotetext{
19 "When the larger narrative alludes to its status as fiction it does so without compromising the emotional integrity of the individual castaways and their memories. They are not aware of being characters, or vehicles for larger themes. [...] There is, then, a tension between the foregrounding of the structural aspects of Lost and the maintenance of the 'fourth wall' within the story itself », Michelle Lang, op. cit.

${ }^{20}$ Voir Mittell, op. cit., p. 130.
} 